\title{
Carnets
}

Revue électronique d'études françaises de l'APEF

Deuxième série - 21 | 2021

Arts du vin

\section{Voyage aux confins du monde avec le vin}

\section{João Domingues}

\section{(2) OpenEdition}

\section{Journals}

Édition électronique

URL : https://journals.openedition.org/carnets/12970

DOI : 10.4000/carnets. 12970

ISSN : 1646-7698

Éditeur

APEF

\section{Référence électronique}

João Domingues, «Voyage aux confins du monde avec le vin », Carnets [En ligne], Deuxième série - 21 | 2021, mis en ligne le 31 mai 2021, consulté le 01 juin 2021. URL : http://journals.openedition.org/ carnets/12970 ; DOI : https://doi.org/10.4000/carnets.12970

Ce document a été généré automatiquement le 1 juin 2021.

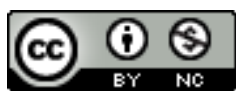

Carnets est mis à disposition selon les termes de la licence Creative Commons - Atribution - Pas d'utilisation commerciale 4.0 International. 


\title{
Voyage aux confins du monde avec le vin
}

\author{
João Domingues
}

\section{Introduction}

1 «L'histoire du vin, n'est-ce pas l'histoire du monde ? ", s'interrogeait déjà au deuxième siècle de notre ère, Babrius² ${ }^{2}$ fabuliste probablement né en Syrie, romain hellénisé ou de langue grecque. La culture de la vigne fut certainement précédée par la vigne sauvage, espèce de liane forestière, très répandue, aujourd'hui encore, de la mer Caspienne jusqu'à l'Atlantique, surtout en région méditerranéenne. Et comme elle appartient au groupe de plantes qui ont vite reconquis une grande partie de l'Europe après la dernière glaciation (entre 110.000 et 10.000 av. JC.), on peut dire que la vigne y a sûrement précédé la présence de l'homme. La vigne fut cultivée d'abord en Egypte et en Phénicie il y a plus de trois mille ans avant notre ère, de deux mille ans en Grèce, de mille ans en Italie, en Sicile et en Afrique du Nord, puis en Espagne, au Portugal et dans le Sud de la France. Pendant les premiers siècles de notre ère, elle fut également introduite, par les Romains, dans le nord de l'Europe et en Grande-Bretagne.

2 Quant à la vinification, elle a sûrement devancé la culture de la vigne, l'homme du néolithique (entre 7.000 et $2.500 \mathrm{av}$. JC.) ayant très bien pu gouter du vin fait à partir du jus fermenté des raisins cueillis sur des vignes sauvages (Charvet et alii, 2007). D'après une étude publiée en 2017, des jarres trouvées dans les environs de Tbilisi indiquent que le 'berceau' du vin se situerait en Géorgie, et que la fabrication de vin remonterait à près de huit mille ans avant notre ère. La découverte d'autres témoignages et d'outils, de la fin du quatrième millénaire, montre que, plus tard, la production de vin s'est répandue de Géorgie à Abydos en Egypte, en passant par l'Arménie, la Turquie, la Palestine, Israël ou la Jordanie. Toutefois des installations récemment mises à jour en Arménie, destinées à presser les raisins, dateraient du début $\mathrm{du} \mathrm{vI}^{\mathrm{e}}$ millénaire ; ce même genre d'installation aurait été utilisé jusqu'au $\mathrm{xIX}^{\mathrm{e}}$ siècle dans tout le bassin méditerranéen ${ }^{3}$. 
3 D'après les études de Lang publiées en 1992 sur la flore postglaciaire, la vigne et les vins de Palestine et d'Israël furent suffisamment connus pour faire l'objet d'exportations dès la fin du quatrième millénaire. Ce qui nous permet de mieux comprendre des expressions qui ont presque trois mille ans, comme celle que l'on trouve dans le Livre d'Osée dans l'Ancien Testament où il est dit, à propos du peuple d'Israël : "Ils feront fleurir la vigne qui aura la renommée du vin du Liban » (Le livre d'Osée, $14: 8)^{4}$.

Ce faisant, le prophète Osée, bien qu'appartenant à une époque de turbulence du VIII siècle avant notre ère, veut annoncer un bel avenir au peuple d'Israël. La vigne et le vin y seraient donc des réalités abondamment connues et reconnues. Osée, le prophète du rapport affectif entre Dieu et son peuple, qui décrit l'égarement du peuple en termes d'infidélité - telle une femme infidèle, à qui le mari pardonne afin qu'elle retombe dans ses bras -, choisit l'image de la vigne et du vin pour souligner combien ce peuple, revenu dans les bras de son Dieu, sera grand, fleurira et acquerra une renommée qui couvrira l'univers ... à l'image de la vigne et du vin.

On sait que, lors des cérémonies religieuses juives, le vin était abondant: pendant la cène, par exemple, qui rappelait la veille de la sortie d'Égypte, on boit du vin au moins à quatre moments différents, toujours accompagnés de prières de remerciements à Dieu. Le vin y symbolise la joie de la libération, la réussite de la Pâque (du passage), et de l'arrivée en terre promise, ainsi que l'espoir d'une nouvelle vie heureuse. Par ailleurs, il est très significatif que, pour encourager le peuple à entrer dans la terre promise à leurs ancêtres, Caleb - parti explorer la région avant l'arrivée du peuple élu -, ramène, entre autres produits, une grappe de raisin, si grosse qu'il a fallu deux hommes pour la porter pendue à une perche ${ }^{5}$. Cette grappe de raisin de Caleb est toujours représentée, comme on le sait, sur la pièce de monnaie israélienne, le Sheckel.

6 En Égypte, le procédé de vinification, avec ses scènes de pressurage et de vendange, apparait représenté depuis le troisième millénaire avant notre ère sur des bas-reliefs, ce qui tend à en confirmer la présence, même si, selon certaines études, le vin y était une boisson rare et réservée aux classes supérieures. Étrangère à la flore égyptienne, la vigne y fut probablement introduite à partir de Canaan ; la production de vin requérait une attention particulière, et sa consommation révélait, naturellement, un certain prestige (Guash-Jané et alii, 2005).

7 En Grèce, depuis l'Antiquité la terre du bon vin par excellence, on trouve déjà des instructions assez précises sur le vin et sa fabrication; la description d'Hésiode, par exemple, est à la fois poétique et précise :

Quand Orion et Sirius auront atteint le milieu du ciel et qu'Aurore aux doigts de rose pourra voir Arcturus, alors, Persès, cueille et rapporte chez toi toutes les grappes. Expose-les au soleil dix jours et dix nuits, mets-les à l'ombre pendant cinq. Le sixième jour, puise et mets dans des vases les dons de Dionysos riches en joies ${ }^{6}$.

8 Ici, Hésiode, poète $\mathrm{du}$ vIII ${ }^{\mathrm{e}}$ siècle avant notre ère, décrit la fabrication du vin, qu'il appelle «les dons de Dionysos riches en joies », ce qui nous laisse deviner quel était l'effet, en tout cas le plus connu, de ces dons dionysiaques chez l'homme.

Encore sur l'histoire du vin, un dernier mot sur le mot lui-même : en grec, önós, qui par l'intermédiaire de l'étrusque a donné vinum en latin, est sûrement d'origine indoeuropéenne ; la même racine est présente en mésopotamien (akkadien), en hittite et en mycénien, en ougaritique et en hébreu. Son origine se situe, très probablement, entre l'Anatolie et le Caucase où, justement, sur les pentes du Mont Ararat, la Bible fait Noé planter ses premières vignes, juste après le déluge (Genèse, VIII: 4$)^{7}$. Le récit précise 
même que Noé y « commença de planter la vigne » et en but le vin (Genèse, Ix : 20-21) ${ }^{8}$. Ce chapitre IX du Livre de la Genèse se termine sur la précision que, après le déluge, Noé avait encore vécu trois-cent-cinquante ans. Il aurait donc vécu en tout et pour tout neuf-cent-cinquante! Rien ne le précise, mais on peut supposer que sa longévité se devrait aussi à ce nectar produit sur les monts d'Ararat.

Ainsi, notre 'histoire française' de Grecs phocéens qui introduisirent en Gaule la culture de la vigne au vi ${ }^{e}$ siècle avant notre ère, et d'Étrusques venus commercialiser leurs vins à Massalia (Marseille), premier comptoir phocéen de la même époque, est de fait une histoire très récente. Car, à cette époque, la vigne et le vin avaient déjà parcouru un long chemin, leurs origines se confondant presque avec celles de l'humanité.

\section{Le vin dans la mythologie}

10 L'Histoire nous montre souvent que toutes les grandes expériences humaines furent immortalisées dans la mythologie ancienne ; celle du vin n'y échappe pas : Dionysos et Priape, son fils; Bacchus et les Bacchantes, Ampélos, le jeune compagnon de Dionysos, Ganymède et Hébé ou Juventus; enfin l'ambroisie et le Nectar, breuvages des immortels.

D'après une légende, la vigne fut le cadeau du dieu Dionysos au roi Oenée qui avait accepté de lui prêter sa femme (Schmidt, 1986 : 315) : le plus grand don - le vin - pour récompenser la plus grande faveur qu'un homme puisse faire à un dieu, à l'époque où les dieux et les hommes cohabitaient sur terre.

Une autre version raconte que c'est Staphylos (qui veut dire grappe, en grec), le berger $\mathrm{du}$ roi qui, voyant un jour une de ses chèvres cabrioler de joie, après avoir mangé du raisin, imagina, en conséquence, de presser ce fruit et d'en recueillir ce jus qui est devenu le vin. Oeunopion, roi de Quios, l'introduisit dans son royaume; et Saturne enseigna, à son tour, la culture de la vigne aux Romains (Schmidt, 1986 :316).

11 Mais Dionysos, dieu du vin, est un fils illégitime de Zeus, le père des dieux; et il fut persécuté dès sa naissance et obligé de fuir dans de lointains pays; il fut même changé en chevreau (cette jeune nature toujours un peu ivre à vrai dire...) pour échapper aux persécutions; des nymphes le prirent en pitié et s'occupèrent de son éducation. Cependant, arrivant à l'âge adulte, il fut frappé de démence et repartit errant dans le monde entier. Grand dieu, mais toujours ivre et souvent très fatigué, il introduisit, dans chaque pays de son errance, la culture de la vigne et la fabrication du vin. C'est ainsi que, selon la légende, on le vit parcourir l'Égypte, la Syrie, la Phrygie et la Thrace. Et après avoir établi un culte dans tous les pays de la Méditerranée, ses compagnons de route et lui - Silènes, Satyres et Bacchantes - partirent pour l'Inde. Revenu en Grèce, il implanta son culte en Béotie, puis navigua vers Naxos, sa dernière demeure, où il imposa aussi son culte avant d'être reçu, de plein droit, dans l'assemblée des dieux dans l'olympe.

12 En Grèce, la culture de la vigne et le culte de Dionysos furent étendus partout, ce dieu étant devenu aussi un dieu des jardins et des bois, symbole de cette force pure, joviale et créatrice de la nature : telle la sève qui gonfle les grains de raisin, ainsi la vie même dans toute la végétation. Aussi, entouré des Bacchantes, ce dieu prend les traits d'un dieu de la 'vie joyeuse', des jeux et des fêtes, surtout dans l'Empire romain, où il prit le nom de Bacchus. Enfin, en tant que dieu de la création et de la créativité - et par conséquent des arts aussi-, il prend l'allure d'un dieu jeune, "généralement 
accompagné par des cortèges (...) de joueurs de flûte qui (...) se livrent à des jeux, à des danses frénétiques et à des transports désordonnés " (Schmidt, 1986: 101). C'est pourquoi, dans tout acte créateur, il y aura toujours cette impulsion première, ce premier jet pur, brut, sauvage, dionysiaque.

Toujours à l'époque où les dieux côtoyaient les humains sur terre, Ampélos (qui veut dire 'cep de vigne'), compagnon de Dionysos, tomba des branches d'un orme couvert de pampres sur lequel il avait grimpé pour cueillir des grappes de raisin; or, Dionysos, extrêmement touché par le sort de son jeune compagnon, pria pour lui, et à sa prière, Ampélos fut placé au ciel au nombre des constellations (Schmidt, 1986:36); telle était l'importance de son acte, ou l'importance de l'amitié créée autour du vin.

Chez les Hittites, quelques mille-six-cents ans avant notre ère, où la vigne était symbole de vitalité et de fécondité, l'arbre s'appelait simplement "arbre de vin», mais qui signifiait aussi « arbre de vie ", comme s'ils voulaient déjà préfigurer la dualité vin / vie que l'on trouve aussi bien dans les mythes dionysiaques que dans la religion chrétienne.

Enfin, le vin entra dans l'olympe, où le beau Ganymède - originaire de l'Asie Mineure, arraché à la terre par Zeus et ravi dans les cieux -, sert d'échanson lors de l'assemblée des dieux, à qui il verse de l'ambroisie, qui rend immortel, et du nectar pour les désaltérer.

\section{Le vin et l'imaginaire}

Tout au long de l'Antiquité, le vin était naturellement omniprésent dans la littérature grecque et nourrit une panoplie de récits dont de nombreux aspects relèvent d'une fonction civilisatrice. Dionysos, par exemple, enseigne l'art de faire du vin aux Grecs, mais Amphictyon leur ordonne de le couper avec de l'eau pour atténuer les mauvais effets de l'alcool. Devenu l'un des trois piliers de la triade méditerranéenne - céréalesolivier-vigne -, le vin est présent dans le quotidien des Grecs, mais aussi dans leur imagination. Moins sacralisé et plus proche du simple produit de consommation, le vin y est l'objet de mille et une histoires dans lesquelles il manifeste son pouvoir, et parfois ses effets pervers :

Rappelons tout d'abord, bien que très brièvement, l'histoire du Cyclope Polyphème, à qui Ulysse fit boire du vin. La potion, aussi précieuse qu'irrésistible, endormit le monstre, et Ulysse put, par le truchement du vin, maitriser son ennemi et libérer ses compagnons.

17 Dans un récit beaucoup plus tardif, en Afrique du nord, Apulée de Madaure ${ }^{9}$ raconte dans ses Métamorphoses l'histoire des brigands qui avaient ravi une jeune fille. Emprisonnée dans un antre enfoui dans la forêt, et ayant perdu tout espoir de survivre, elle fut pourtant libérée par son jeune fiancé, aidé en cela par ce miraculeux allié, le vin. En effet, arrivé au campement des malfaiteurs et faisant semblant d'être lui-même un brigand, il apporta aux ravisseurs des outres de vin pour fêter, disait-il, leurs derniers exploits. Pour gagner leur confiance, il leur versa «à les en noyer tous, de larges coupes, l'une sur l'autre » (Apulée, Livre 7, chap. XI : 16) et les enivra tous. Puis, réconfortés, sa fiancée et lui, par quelques coupes de vin « qu'il commençait par porter à ses lèvres " (ibidem: 16), pendant que les brigands dormaient profondément «noyés dans une crapuleuse ivresse du vin non mélangé cette fois, mais légèrement tiédi (...), ensevelis dans le vin, tous ivres morts pareillement » (ibidem : 17) -, le jeune 
couple s'évade et recouvre la liberté. Or, dans ce récit très allégorique, la jeune fille s'appelait Charité et son fiancé Tlépolème. En vérité, l'industrie d'un jeune fiancé - si ressemblant soit-il au célèbre guerrier achéen $\mathrm{T} \lambda \eta \pi$ ${ }^{\prime} \lambda \varepsilon \mu \circ$ (Tlêpólèmos), voire au fils d'Héraclès du même nom et grand guerrier troyen - n'aurait servi à rien sans la double présence de la puissance du vin. Car c'est le vin, ou par le vin, à la fois bourreau pour les brigands et source de courage pour le jeune couple, que cette Charitas - qu'elle soit la grâce, la gaieté, la gratuité ou la beauté qui répand la joie aussi bien dans la nature que dans le cœur de l'homme - retrouve sa liberté.

L'histoire que nous raconte le grec Lucien de Samosate, dans son Histoire véritable, à propos de «L'Ile du vin » (Lucien, 1958 : 1347-1348) est bien plus fantaisiste, mais non moins symbolique. Lucien vient de partir, sans but précis, pour un grand voyage sur les mers avec cinquante compagnons, tous aussi curieux que lui. « La cause de mon voyage, dit-il, et son intention étaient l'activité de mon esprit et mon désir de choses nouvelles » (ibidem : 1346). Emportés par le vent pendant soixante-dix-neuf jours, ils abordèrent l'île du vin. Les fleuves y étaient de vin et avaient comme source d'énormes vignes d'où coulaient des filets clairs de vin pur. Ils y pêchèrent des poissons qui les enivrèrent lors de la dégustation (ibidem : 1347-1348), et quelque chose de prodigieux se produit alors : « en fait de vigne (...), à partir de la terre, la souche était vigoureuse et forte, mais, au-dessus, c'étaient des femmes, parfaitement formées " (ibidem : 1348). De l'extrémité de leurs doigts et de leurs têtes jaillissait une profusion de vrilles, de feuilles et de grappes. Elles parlaient le Grec, le Lydien et même la langue indienne. Ceux des compagnons de Lucien qu'elles embrassèrent sur la bouche, plongèrent immédiatement dans un état d'ivresse. D'autres, voulant s'unir à elles, s'enracinèrent aussitôt et devinrent comme elles. Le vin séducteur, en imagination ou pour de vrai, peut donc nous rendre prisonniers et faire de nos rêves des cauchemars.

Le vin n'est donc pas toujours cet ellébore bienfaiteur qu'ils ont découvert au cours de leur long voyage, dans l'île des Bienheureux où ils furent reçus par une brise embaumée de parfums de rose, de lys et de violettes, de myrrhe, de laurier et de vigne en fleur (ibidem : 1367), où ils ont assisté à un vrai miracle philosophique : en effet, ils ont vu de leurs yeux vu, Diogène de Sinope, le cynique des cyniques, changer de manières et danser, se livrer à toutes sortes d'excentricités, tomber amoureux et se marier à Laïs, la courtisane des courtisanes ... "quand il était dans son vin» (ibidem: 1371), évidemment.

Ce voyage, au-delà du monde connu, abordant des sujets souvent étranges, est rempli de choses incroyables, bien que présentées de façon très vraisemblable. Mais le narrateur avait bien précisé, dès le début de son histoire, qu'il n'allait rien dire de véritable, et qu'il avait décidé de mentir, «mais avec plus d'honnêteté que les autres, car il est un point sur lequel je dirai la vérité, dit-il, c'est que je raconte des mensonges. (...) Aussi, mes lecteurs doivent-ils ne leur ajouter aucune créance » (ibidem : 1346).

Or, dans ces Histoires véritables - où le titre même est un mensonge -, et malgré toute cette fantaisie, le fruit le plus pur de l'imagination, le lecteur peut pourtant entrevoir le désir d'aller au-delà, et d'en savoir plus. Car de Lucien de Samosate à Voltaire, en passant par Rabelais, cette ébriété fantaisiste n'est souvent qu'une allégorie d'une autre soif qui demande à être désaltérée, dans d'autres mondes, par d'autres breuvages, et dont le vin n'est que l'émule terrestre le plus connu. 


\section{Le vin guérit} voulant se suicider, fut guérie après avoir bu de ce liquide considéré comme un poison en raison de son étrange odeur ; cette légende expliquerait, que, en Iran, on l'appelle «le poison agréable» (Zeher-i-khos; cf. Charvet et alii, 2007). Et Hippocrate, toujours occupé de la santé, d'ajouter que : «Le vin est chose merveilleusement appropriée à l'homme si, en santé comme en maladie, on le boit avec à-propos et juste mesure, suivant la constitution individuelle $»^{10}$.

Quelques années plus tard, Théophraste (372-287 av. J.C) invente les vins médicinaux en y faisant macérer des herbes et des épices.

De la Bible, où le vin est cité des centaines de fois pour ses bienfaits, retenons juste l'histoire du bon Samaritain qui prend soin d'une victime abandonnée sur le bord de la route en lui versant de l'huile et du vin sur les plaies (Luc, $\mathrm{x}: 34$ ).

$\mathrm{Au}$ Moyen Âge, le célèbre Liber de vinis / Le livre des vins ${ }^{11}$ - manuscrit latin des années 1322 (Guerrero, 2013) -, renouant avec la tradition pharmacologique gréco-latine enrichie par la médecine arabe, est un recueil de recettes de vins médicinaux où le vin, en soi déjà considéré comme un agent thérapeutique, sert en outre, par fermentation ou par décoction, à révéler les substances médicinales et à rendre plus agréable la prise de médicaments. C'était apparemment la composition idéale de l'excipient, déjà dûment aromatisé à l'époque.

Mille ans après Hippocrate, Louis Pasteur, soucieux de l'hygiène publique, et sachant que le vin est exempt de microorganismes pathogènes pour l'homme, reprend, presque ipsis verbis, les mots du père de la médecine, en faisant observer que «le vin peut être à bon droit considéré comme la plus saine, la plus hygiénique des boissons » (Pasteur, $1866: 56)^{12}$.

Le vin soulage aussi l'esprit. L'adage peut-être le plus connu sur les effets du vin, c'est que « le vin noie les soucis » de l'homme ; comme le disait Anacréon ${ }^{13}$ au sixième siècle avant notre ère, lui le chanteur de l'amour par excellence, moins maître à penser que maître à vivre!

Pétrone ${ }^{14}$, un autre maître à vivre, nous offre sa philosophie de vie, bien imbibée de vin dans sa satire romanesque, le Satiricon. Dans la scène du "Banquet chez Trimalcion $~^{15}$, la plus longue du roman, et évidente parodie du "Banquet de Platon », il est question d'un repas 'philosophique', bien arrosé. En effet, animant les conversations et les discours, le vin y coule à flots, et fait l'objet des réflexions les plus inattendues. Par exemple, en voyant arriver "des amphores de verre, soigneusement scellées", Trimalcion lit sur l'étiquette "Falerne Opimien, de cent ans », et s'exclame aussitôt : « Las, [...], ce vin, il a donc vécu plus longtemps que le chétif humain! Aussi, allons-y à gogo. La vie c'est le vin» (Pétrone, Le Satiricon, XXXIV : 28).

Au miel ou coupé d'eau chaude, ce nectar réchauffe l'esprit et le corps, car, comme l'on dit, depuis au moins le deuxième siècle, "le boire chaud c'est le meilleur des manteaux » (ibidem, XLI : 33). Comme chez Platon, ce banquet est saturé de discours et de réflexions; et comme Alcibiade, l'invité de Socrate au 'Banquet', le sculpteur Habinnas arrive chez Trimalcion déjà éméché (ibidem, LXV : 54) et d'humeur enjouée ; il va sans dire qu'avant même de commencer son intervention, il réclame « du vin et de l'eau chaude » (ibidem, LXV : 55).

$\mathrm{Au}$ cours du banquet, l'alchimie du vin atteint son paroxysme lorsque le richissime

Carnets, Deuxième série - 21 | 2021 
Trimalcion, blessé par un esclave maladroit, décide incontinent, non seulement de lui pardonner, mais de l'affranchir pour que l'on ne puisse dire qu'il avait été offensé par un inférieur; plus tard, il invite même certains esclaves à se mettre à table avec ses convives (ibidem, LXX : 59) ; puis, suite à autre désordre, survenu au beau milieu du banquet, et dans la bonne humeur, il « fit préparer un grand vase de vin (...) et ordonna de donner à boire à tous les esclaves » (ibidem, LXIV : 54), sans faire de distinction avec ses invités. Trimalcion proclame ainsi une fraternité humaine universelle, qu'il ne cessera de renforcer, tantôt en rappelant à ses convives que tous les hommes esclaves, affranchis ou hommes libres -, sont nés de la même façon, tantôt en faisant remarquer que tous ont tété le même lait. Il finira même par affranchir tous ses esclaves dans son testament et par leur léguer la plupart de ses biens ${ }^{16}$. Béni donc soit le vin qui déclenche de tels incidents et fait naître chez l'homme de telles idées.

Héritier de Lucien, de Pétrone ou d'Érasme, François Rabelais, homme tolérant et grand humaniste, mariant la parodie et la satire pour s'en prendre aux abus des grands, propose au lecteur la franchise et la banalité d'une culture populaire, apparemment faite de jeux et de vins, mais en vérité pétrie de valeurs ; car, chez lui, même le vin est le passeur de leçons pour les hommes. Il suffira, pour s'en convaincre, de se rappeler le prologue de Gargantua, dans lequel il s'adresse aux «Buveurs très illustres » (Rabelais, $1929^{\mathrm{a}}: 3$ ), seuls capables de "sentir et estimer ces beaux livres de haute graisse », puis, par attentive lecture, de "rompre l'os et sucer la substantifique moelle» (Rabelais, $1929^{\mathrm{a}}:$ 5). De fait, dans l'Abbaye de Thélème (Rabelais, $1929^{\mathrm{a}}: 189-190$ ), non seulement on boit du vin, mais on discute joyeusement.

De même, l'oracle caché dans la 'Dive Bouteille' (Cinquième Livre, chap. XLIV) était Trinch, c'est-à-dire 'bois', cherche par toi-même le savoir, et jouis-en, par toi-même, en pleine liberté! Pour étancher la soif de savoir, pour nourrir l'ébriété créatrice, «buvez », donc, vous dit-on, car dans ce vin-là est cachée la vérité. " La Dive Bouteille vous y envoie, soyez vous-mêmes interprètes de votre entreprise " (Cinquième Livre, $\mathrm{XLV}: 169)^{17}$. Il n'est pas difficile de reconnaitre dans ce passage une paraphrase évidente du « connais-toi toi-même » ( $\Gamma \nu \tilde{\omega} \theta_{\imath} \sigma^{\prime} \alpha u \tau o ́ v$, en grec) de Socrate.

\section{IV - Le vin dans la religion}

21 Enfin, dans ce survol à la fois chronologique, géographique et thématique, je ferai une dernière référence au vin, au vin dans la religion. "Point de fête sans vin », dit un proverbe juif. Or, si le vin occupait déjà une place de choix dans la culture du peuple d'Israël, le christianisme lui ajoute un sens nouveau, celui de l'alliance d'Amitié indestructible entre Dieu et l'Homme. Parmi les quatre évangélistes, trois racontent le moment où Jésus prend la coupe et partage le vin, invitant ses disciples à perpétuer ce geste en mémoire de Lui ${ }^{18}$. Jean, l'évangéliste de l'amour, ne décrit pas le rite de la coupe et du vin et le remplace par un autre bien différent, mais qui a précisément le même sens ${ }^{19}$ : Jésus lave les pieds de ses disciples et leur dit: je vous ai donné l'exemple; «aimez-vous les uns les autres », et ainsi le monde "saura que vous êtes mes disciples » (Jean, XIII : 25).

La coupe utilisée par le Christ lors de la Cène était sûrement un crater, une grande coupe, d'un litre ou deux, dont le vin sera partagé avec les douze disciples, avec sa famille et d'autres amis, réunis pour célébrer la Pâque ${ }^{20}$ avec lui. La valeur symbolique réside dans le geste du « boire ensemble » avec Jésus, autrement dit partager ses choix, 
sa façon de voir la vie et le monde, sa façon de vivre. Le Christ scellera, à l'extrême, cette nouvelle alliance avec son sang, sur la croix. Mais affirmer que, dans la messe, le contenu de la coupe se transforme en sang du corps de Jésus, n'est, pour les théologiens actuels, qu'une 'grossièreté médiévale ${ }^{21}$, introduite par ceux qui avaient oublié la valeur symbolique du geste du Christ.

Ainsi, si au long de l'histoire de l'Église et dans les différentes églises, notamment les églises orientales, on a bien continué d'utiliser le vin lors de ce rite (normalement du bon vin, qui pouvait mieux se conserver), dans certaines régions chinoises, on prenait du 'vin de riz'22; et jusqu'à très récemment, au Soudan par exemple, les missionnaires catholiques, faute de vin, recouraient à la fermentation de raisins secs dans de l'eau. En fait, utiliser 'le même vin' et répéter 'le même geste' sert davantage à traduire cette fraternité universelle et à célébrer 'la même mémoire', où que l'on soit sur terre ${ }^{23}$.

\section{Conclusion}

Aux origines perdues au fin fond de l'Histoire, le vin n'est donc pas un simple produit, une simple boisson; entre mythe et histoire, le vin matérialise et traduit une très longue expérience humaine et beaucoup de savoir. Son histoire se confond avec l'histoire de l'Humanité; sa mythologie recense et reflète souvent, en guise de problématique existentielle, beaucoup de l'expérience humaine sur terre.

Enfin, le rapport du vin à la soif de savoir humain sous-tend, comme on l'a dit, un rapport métaphorique, l'un comme l'autre rendant consubstantiels le corps et l'esprit : soin et baume pour le corps souffrant, ou élixir pour l'esprit qui peut y noyer ses peines; dans les rapports intimes ou dans la vie en société, il est toujours là, à tout moment, pour le partage et le réconfort.

\section{BIBLIOGRAPHIE}

APULÉE (1985). Les Métamorphoses, t. 3, Livre VII. Paris : Les Belles Lettres.

BRUN, Jean-Pierre (2003). Le Vin et l'huile dans la Méditerranée antique : viticulture, oléiculture et

procédés de fabrication. s.l. : Éditions Errance.

BRUN, Jean-Pierre (2004). Archéologie du vin et de l'huile. De la préhistoire à l'époque hellénistique. s.l. :

Éditions Errance.

BOUVIER, Michel (2009). Le vin, c'est toute une histoire. Paris : Jean-Paul Rocher Éditeur.

CHARVET, Pascal, ARGOD-DUTARD, Françoise et LAVAUD, Sandrine (2007). Voyage aux pays du vin : des

origines à nos jours. Histoire, anthologie et dictionnaire. s.l. : Robert Laffont.

DION, Roger (1959). Histoire de la vigne et du vin en France des origines au XIX siècle. Paris :

Flammarion.

GARRIER, Gilbert (1995). Histoire sociale et culturelle du vin. Paris : Bordas Cultures. 
GAUTIER, Jean-François (1992). Histoire du vin. Paris : PUF, collection « Que sais-je », nº 2676.

GAUTIER, Jean-François (2003). Le Vin de la mythologie à l'œnologie, L'esprit d'une civilisation.

Bordeaux : Édition Féret.

JABOULET-VERCHERRE, Azélina (2012). Florilège de Discours savants sur le vin. Bordeaux : Féret.

JABOULET-VERCHERRE, Azélina (2016). Le Vin, entre nature et culture. Bordeaux : Féret.

JOHNSON, Hugh (2002). Une histoire mondiale du vin. Paris : Hachette Pratique.

LUCIEN DE SAMOSATE (1958). Histoire véritable, in Romans grecs et latins, textes présentés, traduits et annotés par Pierre Grimal. Paris : Éditions Gallimard, coll. «Bibliothèque de la Pléiade », pp. 1339-1384.

MCGOVERN, Patrick E. (2007). Ancient Wine: The Search for the Origins of Viniculture. Oxford: Princeton University Press.

NESTLE, Erwin, ALAND, Kurt (196422). Novum testamentum, Graece et Latine, Stuttgart,

Wurttembergische Bebelanstalt.

NOURRISSON, Didier (2017). Histoire du vin. S. 1. : Éditions Perrin.

PASTEUR, Louis (1866). Études sur le vin, ses maladies, causes qui les provoquent, procédés nouveaux pour le conserver et pour le vieillir. Paris : À l'Imprimerie Impériale.

PÉTRONE (1958). Le Satiricon, in Romans grecs et latins, textes présentés, traduits et annotés par Pierre Grimal. Paris : Éditions Gallimard, coll. « Bibliothèque de la Pléiade », pp. 7-137.

POUX, Mathieu, DIETLER, Michael (2004). Le Vin, nectar des dieux, génie des hommes. s.l. : Éditions Infolio.

RABELAIS, François (1929a). Gargantua. Paris : Édition Fernand Roches.

RABELAIS, François $\left(1929^{b}\right)$. Le Quart Livre. Paris : Édition Fernand Roches.

RABELAIS, François $\left(1929^{c}\right)$. Le Cinquième livre. Paris : Édition Fernand Roches.

RIVES, Max (1986). « Les Origines du vignoble », in La Vigne et le vin, numéro hors-série trimestriel de Science \& Vie, $\mathrm{n}^{\circ} 155$, septembre 1986, pp. 12-19.

SCHMIDT, Joël (1986). Dictionnaire de la mythologie grecque et romaine. Paris : Librairie Larousse.

VILLENEUVE, Arnaud de (1322 ? - 2011). Le livre des Vins, traduit du latin, préfacé et annoté par Patrick Gifreu. s.l. : Éditions de la Merci.

\section{NOTES}

1. Bonum vinum laetificat cor hominis / Le bon vin réjouit le cœur de l'homme (Livre de l'Ecclésiaste, $\mathrm{XL}: 20)$.

2. Babrius, peut-être Valerius Babrius, fabuliste romain hellénisé du II $^{\mathrm{e}}$ siècle. Il a réécrit les fables d'Ésope. Son texte, un manuscrit renfermant 123 fables, perdu pendant des siècles, fut retrouvé en 1843, au mont Athos.

3. «Une unité complète de production de vin datant de 6100 ans, la plus ancienne connue à ce jour, a été découverte dans une caverne en Arménie, a annoncé aujourd'hui une équipe internationale d'archéologues. Avant cela, les vestiges comparables d'équipement de production viticole remontaient à 5000 ans. "Pour la première fois, nous avons une image archéologique complète d'une production de vin vieille de 6100 ans", se félicite Gregory Areshian, responsable 
des fouilles et directeur adjoint de l'Institut d'Archéologie Cotsen à l'Université de Californie à Los Angeles. Parmi les objets mis au jour figurent des pépins de raisin, des restes de raisins pressés, des sarments de vigne atrophiés, un pressoir rudimentaire, une cuve en argile apparemment utilisée pour la fermentation, des tessons de poterie imprégnés de vin, et même une tasse et un bol pour boire» (in: http://www.lefigaro.fr/flash-actu/ 2011/01/11/97001-20110111FILWWW00423-vin-une-unite-de-production-de-6100-ans.php [consulté le 26 juin 2019].

4. Toutes les références bibliques renvoient à la Bible dite 'de Jérusalem'.

5. Le Livre des Nombres, XIII, 23-24 : « ${ }^{23}$ Ils parvinrent au val d'Eshkol ; ils y coupèrent un sarment et une grappe de raisin qu'ils emportèrent à deux, sur une perche, ainsi que des grenades et des figues. ${ }^{24}$ On appela ce lieu val d'Eshkol, à cause de la grappe qu'y avaient coupée les Israélites ».

6. Hésiode, Des Travaux et des Jours, 609-614.

7. Livre de la Genèse, VIII : 4 : « et, au septième mois, au dix-septième jour du mois, l'arche s'arrêta sur les monts d'Ararat $"$.

8. Livre de la Genèse, IX : 20-21 : « Noé, le cultivateur, commença de planter la vigne. Ayant bu du vin, il fut enivré et se dénuda à l'intérieur de sa tente ».

9. Lucius Apuleius (en Latin) est né vers 125 à Madaure, actuelle M'daourouch, située au nord-est de l'Algérie.

10. Cf. : «Les pouvoirs du vin depuis l'Antiquité » (in: http://www.sommelier-on-line.com/ p146.html [consulté le 20 août 2019].

11. Manuscrit daté de 1322 , le Liber de vinis, editus ab Arnaldo de Villa Noua. Laudamus vinum de bona vite ad conficiendum vina medicinalia fut imprimé à Leipzig, sûrement après 1500.

12. «Plus on réfléchira aux causes des maladies des vins, plus on se convaincra que l'art de la vinification, et les soins que l'expérience des siècles a proclamés nécessaires, ont principalement leur raison d'être dans les conditions mêmes de la vie et de la manière d'agir des parasites du vin, de telle sorte que, si l'on pouvait arriver à supprimer par une opération pratique très simple, les causes des altérations spontanées des vins, on pourrait, sans nul doute, fonder un art nouveau de faire le vin beaucoup moins dispendieux que celui qui est suivi depuis si longtemps, bien plus efficace surtout pour supprimer les pertes qu'occasionnent les maladies des vins, très propre par conséquent à l'extension du commerce de cette denrée. Il est désirable que l'on atteigne ce but, car le vin peut être à bon droit considéré comme la plus saine, la plus hygiénique des boissons. Aussi, parmi celles qui lui sont connues aujourd'hui, c'est celle que l'homme recherche de préférence à toutes les autres, si peu que l'occasion lui ait été offerte de s'y habituer ». (Pasteur, $1866: 55-56)$.

13. Poète lyrique grec du $\mathrm{VI}^{\mathrm{e}}$ siècle avant notre ère.

14. Pétrone ou Petronius Niger, écrivain romain, auteur du Satyricon, fut un des favoris de Néron.

15. Le Banquet chez Trimalcion (en latin, cena Trimalchionis), est le plus long récit du roman Satyricon de Pétrone. Cet épisode est normalement interprété comme étant une «fable milésienne ». Trimalcion fut esclave ; puis, ayant été affranchi et devenu extrêmement riche - par son ambition mais aussi par son intelligence -, il exhibe dans le roman un luxe de mauvais goût, type littéraire du parvenu. En effet, son statut d'ancien esclave le sépare toujours de la bonne société. Riches affranchis comme lui, ses invités au banquet composent le cadre de cet épisode qui dépeint l'ascension sociale de ces gens. Le vif intérêt pour la psychologie des situations, et la critique sociale qu'elle sous-entend, est une des originalités de Pétrone.

16. "Mes amis, dit-il, les esclaves aussi sont des êtres humains, et ils ont tété le même lait que nous, même si un destin mauvais les accable. Mais, si je vis, ils ne tarderont pas à boire l'eau de la liberté. Bref, je les affranchis tous par testament. Je lègue aussi à Philargyre une propriété et sa femme ; à Carion une maison de rapport, le montant du vingtième et un lit avec sa literie. [...] Et je rends tout cela public afin que mes valets, dès maintenant, aient pour moi autant d'affection que lorsque je serai mort » (ibidem, LXXI : 60). 
17. Chez Rabelais, les héros expriment en permanence cette soif de vie et de connaissance. Introduits par Bacbuc dans le temple de la « belle fontaine onirique », Pantagruel et ses amis vont boire la liqueur qui en jaillit. Le mot trouvé dans la Dive bouteille est «Trinch» et Bacbuc en donne l'explication suivante : « Trinch est un mot panoraculaire, et compris de toutes nations, et il signifie pour nous : Buvez. (...) Mais ici maintenons que ce n'est pas rire, mais boire, qui est le propre de l'homme; je ne dis pas boire simplement et absolument, car aussi bien boivent les bêtes : je dis boire du vin bon et frais. Notez, amis, que de vin divin on devient, et qu'il n'y a argument aussi sûr, ni d'art de divination moins fallacieux. Vos Académiques l'affirment. (...) Car il a le pouvoir de remplir l'âme de toute vérité, de tout savoir et de toute philosophie. Si vous avez remarqué ce qui est écrit en lettres ioniques sur la porte du temple, vous avez pu comprendre que dans le vin est cachée la vérité. La Dive Bouteille vous y envoie, soyez vousmêmes interprètes de votre entreprise » (Cinquième Livre, Chap. XLV : 168-169).

18. Mathieu XVI : 26-29, Marc XIV : 22-25, Luc XXII : 14-20 ; Paul en parle aussi dans sa première lettre aux Corinthiens ( $1^{\mathrm{e}}$ Corinthiens, XI : 23-29).

19. Jean, le disciple de l'amour, raconte que Jésus à la fin du repas enleva ses vestes, prit une serviette et un bassin et commença à laver les pieds à ses disciples (Jean XIII : 4-5).

20. «Prenez, (...) buvez-en tous » (Math. xxvı : 26-27), dit Jésus à ses amis. La Pâque rappelait la délivrance d'Israël hors d'Egypte. Au début de notre ère, les Juifs ne célébraient plus la Pâque comme lors du départ d'Égypte (à la hâte, debout... cf. Livre de l'Exode, xII : 11). Au contraire, la Pâque était devenue un repas long, se déroulant suivant un rituel bien déterminé. Sur la table, à côté de l'agneau, du pain sans levain et des herbes amères, il y avait des coupes de vin. Le père de famille, après avoir bu et avoir passé la coupe à tous les convives, lisait dans les livres de Moïse des passages se rapportant à la sortie d'Egypte; et on récitait des Psaumes. Toujours en rapport avec le vin, un autre geste très symbolique fut celui du Christ mourant sur la croix qui refusa de boire le vin mixtionné de myrrhe, normalement destiné à atténuer les souffrances des crucifiés en les étourdissant (cf. Mathieu, XXVII : 34).

21. Quant à la doctrine de la Transsubstantiation, elle est définie, depuis le Concile de Trente (1551) comme suit : «Praesentia in Eucharistia est vera, realis, identica, non solum quoad divinitatem; efficitur transsubstantiatione; substantia panis et vini cessant; item natura panis cum elementis suis, manentibus speciebus (accidentibus) sine subjecto» (H. Denzinger (1937). Enchiridion Symbolorum, Defininitionum et declarationum de rebus fidei et morum, (Index Systematicus, XIIf., p. 43 de l'Appendix), Friburgi Briscoviae MCMXXXVII, Herder \& CO., Typographi Editores Pontificii, 1937). Selon la doctrine catholique, et faisant appel à la terminologie aristotélicienne, il y a, donc, une transformation de la «substance » (ce vin devient le Sang du Christ, c'est-à-dire présence réelle de Dieu), mais non des "accidents" (l'odeur, la saveur, la texture, la forme, la couleur) qui demeurent inchangeables. De nos jours, même dans l'Église catholique, nombreux sont ceux qui parlent plutôt de «transsignification » ou «transfinalisation », ce qui les rapproche, en fait, de la doctrine des églises luthériennes et calvinistes qui croient plutôt à la "consubstantiation », ce qui veut dire que le vin demeure inchangeable, mais Dieu devient présent (dans' ce vin), réellement, dans l'Eucharistie.

22. Les communautés chinoises de la région de Changang, pendant la dynastie Tang $\left(\mathrm{VI}^{\mathrm{e}}-\mathrm{IX}^{\mathrm{e}}\right.$ siècles), utilisaient le pain/gâteau de riz et le 'vin de riz'.

23. Dans la liturgie catholique, la communion sous les deux espèces (le pain et le vin) fut pratiquée jusqu'au XIII ${ }^{\mathrm{e}}$ siècle et elle fut le 'moteur' du maintien de la tradition viticole dans le monde chrétien. Puis, la colonisation des nouveaux mondes fut, elle aussi, accompagnée par l'expansion de la vigne. Ainsi, des traces de vignoble sud-africain datent de 1659 et furent attestées dans la province du Cap, où il a sûrement été implanté par les premiers colons. Pareillement, axée sur l'expansion du christianisme, la conquête de toute l'Amérique du Sud s'accompagne du développement de la vigne. En Amérique du Nord, il semblerait que la vigne existait déjà à l'état sauvage ; cependant, certaines congrégations missionnaires tentent de tirer 
du vin de ces lambrusques locales afin de pourvoir aux besoins de l'Eucharistie, mais ces tentatives vinicoles furent abandonnées. Ce n'est qu'au XVIII ${ }^{\mathrm{e}}$ siècle que le vignoble californien s'est développé (avec la présence des moines franciscains). Au Canada, dans la région des Grands Lacs, certaines variétés européennes, qui furent implantées au milieu du XVIII ${ }^{\mathrm{e}}$ siècle, y poussent encore de nos jours.

\section{RÉSUMÉS}

«L'histoire du vin, n'est-ce pas l'histoire du monde? » (Babrius, $\mathrm{II}^{\mathrm{e}}$ siècle). Entre imagination et histoire, le vin est l'un des tout premiers produits à avoir fait le tour du monde. De ses origines, qui restent incertaines à bien des égards, jusqu'à sa mondialisation, la vigne et le vin ont fait l'objet des histoires les plus extravagantes et joué les rôles les plus singuliers. N'en retenant que celles qui se rattachent plus directement à notre culture et à notre histoire (et en partant surtout de récits anciens - Platon, Lucien de Samosate, Pétrone ou Apulée - et de la mythologie grécoromaine et chrétienne), nous essaierons, dans cette étude, de problématiser la portée et le(s) sens possible(s) de ces histoires au fil des siècles ainsi que leurs rapports avec l'expérience du contact avec ce nectar qui « met la joie au cœur $»^{1}$.

«Is the history of wine not the history of the world?» (Babrius, 2nd century). Between imagination and history, wine is one of the first products to have travelled the world. Since its origins, which have remained obscure for several reasons, to its globalization, the vine and wine have been the subject of the most extravagant stories and have played the most peculiar roles. Limiting ourselves to the ones directly related to our culture and history (essentially stemming from ancient narratives - Plato, Lucian of Samosata, Petronius or Apuleius - and both greek, latin and christian mythologies), we have attempted, in this essay, to consider the dimension and the meanings in those stories throughout the ages, as well as their relation to experimenting that nectar which « gladdens the heart » (Ecclesiast, XL: 20).

\section{INDEX}

Keywords : wine, history, mythology, imaginary, religion

Mots-clés : vin, histoire, mythologie, imaginaire, religion

\section{AUTEUR}

\section{JOÃO DOMINGUES}

Université de Coimbra -Centre de Littérature Portugaise jcosta[at]fl.uc.pt 